\title{
A genome-wide approach identifies distinct but overlapping subsets of cellular mRNAs associated with Staufen1- and Staufen2-containing ribonucleoprotein complexes
}

\author{
LUC FURIC, ${ }^{1,2}$ MARJOLAINE MAHER-LAPORTE, ${ }^{1,2}$ and LUC DESGROSEILLERS ${ }^{\mathbf{1}}$ \\ ${ }^{1}$ Département de Biochimie, Université de Montréal, Montréal, Québec, Canada H3C 3J7
}

\begin{abstract}
Messenger RNAs are associated with multiple RNA-binding proteins to form ribonucleoprotein (mRNP) complexes. These proteins are important regulators of the fate of their target mRNAs. In human cells, Staufen1 and Staufen2 proteins, coded by two different genes, are double-stranded RNA-binding proteins involved in several cellular functions including mRNA localization, translation, and decay. Although $51 \%$ identical, these proteins are nevertheless found in different RNA particles. In addition, differential splicing events generate Staufen2 isoforms that only differ at their N-terminal extremities. In this paper, we used a genome-wide approach to identify and compare the mRNA targets of mammalian Staufen proteins. The mRNA content of Staufen mRNPs was identified by probing DNA microarrays with probes derived from mRNAs isolated from immunopurified Staufen-containing complexes following transfection of HEK293T cells with Stau ${ }^{55}$-HA, Stau $2^{59}$-HA, or Stau $2^{62}$-HA expressors. Our results indicate that $7 \%$ and $11 \%$ of the cellular RNAs expressed in HEK293T cells are found in Stau1- and in Stau2containing mRNPs, respectively. A comparison of Stau1- and Stau2-containing mRNAs identifies a relatively low percentage of common mRNAs; the percentage of common mRNAs highly increases when mRNAs in Stau $2^{59}$-HA- and Stau $2^{62}$-containing mRNPs are compared. There is a predominance of $m$ RNAs involved in cell metabolism, transport, transcription, regulation of cell processes, and catalytic activity. All these subsets of mRNAs are mostly distinct from those associated with FMRP or IMP, although some mRNAs overlap. Consistent with a model of post-transcriptionnal gene regulation, our results show that Stau1and Stau2-mRNPs associate with distinct but overlapping sets of cellular mRNAs.
\end{abstract}

Keywords: mRNA localization; RNA-protein interaction; ribonucleoproteins; Staufen; microarray

\section{INTRODUCTION}

The regulation of gene expression at the post-transcriptional level is a mechanism widely used by cells to control protein synthesis with acute precision. In the majority of cases, a sequence or a structure in the mRNA (cis element) is recognized by one or more proteins (trans factors) that assemble together in mRNPs and mediate an effect on mRNA metabolism. This interaction scheme has been shown to be involved in the intracellular localization of

\footnotetext{
${ }^{2}$ These authors contributed equally to this work.

Reprint requests to: Luc DesGroseillers, Département de Biochimie, Université de Montréal, P.O. Box 6128, station Centre Ville Montréal, Québec, Canada H3C 3J7; e-mail: luc.desgroseillers@umontreal.ca; fax: (514) 343-2210.

Article published online ahead of print. Article and publication date are at http://www.rnajournal.org/cgi/doi/10.1261/rna.720308.
}

mRNA (Bashirullah et al. 1998), in translational activation (Vagner et al. 2001) or repression (Chekulaeva et al. 2006), and also in mRNA stabilization and decay (Brennan and Steitz 2001; Chen et al. 2001). Many RNA-binding proteins that are trans factors can each associate with more than one mRNA species in the cell, and it has been postulated that the networks of mRNAs bound by these proteins can serve as post-transcriptional operons (Keene and Tenenbaum 2002).

Staufen is an RNA-binding protein first discovered in Drosophila, where it plays a role in oocyte patterning by localizing oskar and bicoid transcripts to the posterior and anterior poles, respectively (Riechmann and Ephrussi 2001). Staufen is also implicated in the translational derepression of oskar once localized at the posterior pole (Micklem et al. 2000). There are two mammalian homologs of Staufen, termed Stau1 and Stau2, and they both possess 
four copies of the double-stranded RNA-binding domain (dsRBD). Both Stau1 and Stau2 are expressed in the somato-dendritic compartment of neurons, where they form distinct RNA granules visible as puncta in dendrites. Stau1 and Stau2 are also involved in mRNA transport along the microtubule tracts in neuronal dendrites (Kohrmann et al. 1999; Tang et al. 2001; Duchaine et al. 2002; Kanai et al. 2004). More recently, Staul was identified as a central component of a new mRNA decay mechanism termed Staufen-mediated decay (Kim et al. 2005). In addition to functions in RNA localization and decay, Staul can also stimulate translation of repressed messengers containing structured RNA elements in their 5' UTR (Dugre-Brisson et al. 2005).

Both genes produce multiple isoforms that are generated by alternative splicing. The Staul gene generates at least two isoforms of $55 \mathrm{kDa}\left(\mathrm{Stau}_{1}{ }^{55}\right)$ and $63 \mathrm{kDa}\left(\mathrm{Stau}_{1}{ }^{63}\right)$ and one isoform that does not bind mRNA (Staul $1^{\mathrm{i}}$ ). This latter isoform may be a regulator of Staul functions. Staul is found to be mainly associated with tubulo-vesicular structures in the cell as observed by microscopy and also is found to cofractionate with ribosomes and the rough endoplasmic reticulum in a sucrose gradient (Wickham et al. 1999; Duchaine et al. 2002). Similarly, the Stau2 gene generates isoforms of $62 \mathrm{kDa}\left(\mathrm{Stau}_{2}{ }^{62}\right), 59 \mathrm{kDa}\left(\mathrm{Stau}_{2}{ }^{59}\right)$, and $52 \mathrm{kDa}\left(\mathrm{Stau}_{2}{ }^{52}\right.$ ) (Buchner et al. 1999; Duchaine et al. 2002). In a sucrose gradient of neuron extracts, Stau $2^{62}$ is found in ribosome-free light complexes, whereas Stau2 ${ }^{59}$ and $\mathrm{Stau} 2^{52}$ cofractionate with ribosomes.

Overall, Stau1 and Stau2 share $51 \%$ of amino acid sequence identity. When domains involved in mRNA binding (dsRBD3) are compared, the sequence identity between these proteins jumps to $77 \%$ (Fig. 1). The question is whether these highly similar proteins bind overlapping populations of mRNAs or whether they associate with

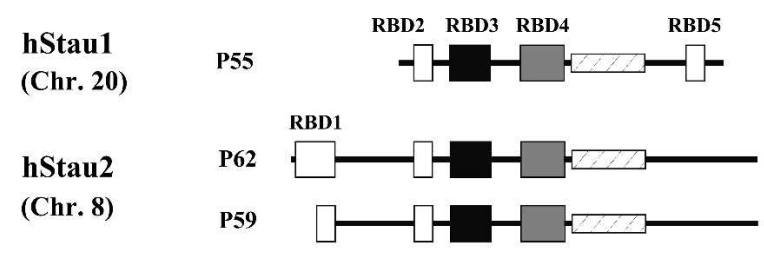

dsRBD3 (sequence identity: $77 \%$ )

Stau1 KSEISQVFEIALKRNLPVNEEVARESGEPHMKNFVTKVSVGEFVEGEGKSKKISKKNAAIAVIEEI Stau2 KSEISLVFEIALKRNMPVSEEVIKESGPPHMKSFVTRVSVGEFSAEGEGNSKKLSKKRAATTVIQEI

FIGURE 1. Comparison of the Staufen paralogs. (Top) Schematic representation of Stau $1^{55}$ (P55), Stau2 ${ }^{59}$ (P59), and Stau2 $2^{62}$ (P62). (Black boxes) Major RNA-binding domains, (gray boxes) minor RNA-binding domains, (white boxes) domains that do not bind RNA even if they show sequence similarity to RNA-binding domains, (hatched boxes) tubulin-binding domain. (RBD) RNA-binding domain, (Chr) chromosome. (Bottom) Sequence comparison of Staul and Stau2 dsRBD3. Amino acids are indicated by the one-letter code. different subsets of mRNAs. The question is even more relevant for alternatively spliced Stau isoforms that are perfectly identical over domains involved in RNA binding. Answering these questions is fundamental for our understanding of Staufen functions in the cells and how they regulate redundant or specific pathways. To date, little is known about the RNA content of Staufen mRNPs. The present work was undertaken in an effort to identify the various mRNAs that are associated with Stau1 and Stau2 in mammalian cells and with differentially spliced Stau2 isoforms. The use of a genome-wide screen using microarrays to identify mRNA subsets associated with RNAbinding proteins is a proven approach that was successfully applied in many studies. This technique, termed RIP-Chip, has been helpful to uncover the identity of the Fragile X mental retardation protein (FMRP) (Brown et al. 2001), YB-1 (Evdokimova et al. 2006), U2AF ${ }^{65}$ (Gama-Carvalho et al. 2006), Insulin-like growth factor II mRNA-binding protein (IMP1) (Jonson et al. 2007), and SLBP (TownleyTilson et al. 2006) copurifying RNAs.

Here, we report the first high-throughput identification of Staufen-associated mRNAs in human HEK293T cells. HA-tagged versions of Stau $1^{55}$, Stau2 $2^{59}$, and Stau ${ }^{62}$ were transfected in HEK293T cells. After immunopurification, the RNA content of Staufen mRNPs was isolated and used to hybridize Affymetrix human gene chips. Hybridizations identified several probe sets with each immunopurified mRNP. A high percentage of them are unique for Staul or Stau2 mRNPs. Enriched mRNAs were classified according to their cellular functions. Gene ontology (GO) annotations suggest that Staufen proteins are mainly involved in the regulation of cellular metabolism and physiological processes.

\section{RESULTS}

\section{Stau1- and Stau2-containing RNP complexes copurify with RNA}

The aim of this study was to identify and compare mRNAs in different populations of Staufen-containing mRNPs as a means to unveil the role(s) of these complexes in the cell regulatory pathways. Since Stau1 and Stau2 were described in different mRNP complexes, they may fulfill differential function by binding to alternative subsets of mRNAs (Duchaine et al. 2002; Thomas et al. 2005). The same may be true for two Stau2 isoforms that were shown to be differentially distributed in cells (Duchaine et al. 2002). Therefore, human embryonic kidney (HEK) 293T cells were transfected with plasmids coding for either Stau $1^{55}$ HA, Stau $2^{59}-\mathrm{HA}$, or Stau $2^{62}$-HA, and Staufen-containing mRNPs were immunoprecipitated using a monoclonal anti-HA antibody for immunoprecipitation (IP). Coimmunoprecipitated mRNAs were isolated from each mRNP extract and used to generate probes in order to hybridize 
microarrays. As a control, immunoprecipitations with antiHA antibodies were done on mock transfected cells. Western blot analysis showed that each Staufen-HA protein and isoform was efficiently immunoprecipitated (Fig. 2A). To determine whether RNA copurified with Staul and Stau2 complexes, RNA was isolated from the immunoprecipitates and visualized using RNA pico chips (Fig. 2B). A clear enrichment of mRNA was observed in samples of immunopurified Staufen-containing RNPs as compared with controls in which a very low amount of copurifying RNA was detected. As expected, mainly two peaks corresponding to $18 \mathrm{~S}$ and $28 \mathrm{~S}$ ribosomal RNA were seen from HEK293T cells total RNA. These data showed that subpopulations of RNA coimmunoprecipitate with each Staufen isoform.

\section{Microarray analysis of Staufen-associated mRNAs}

RNAs isolated from Staufen-HA and mock transfected cells were used as a source of probes to hybridize commercially available human DNA oligonucleotide microarrays from

A

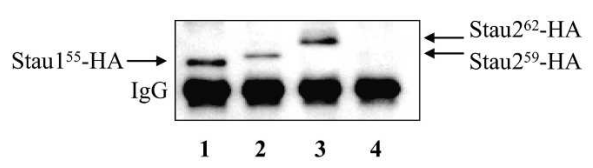

B
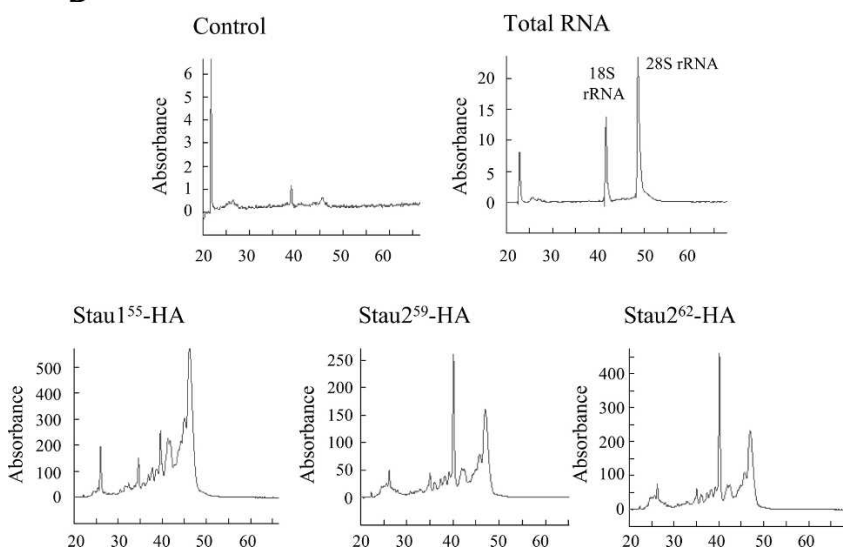

FIGURE 2. Stau1 and Stau2 copurify with RNA. (A) Immunoprecipitation of Staufen-HA constructs. HEK293T cells were either transfected with one of the plasmids expressing Staul ${ }^{55}$-HA, Stau $2^{59}-\mathrm{HA}$, or Stau2 $2^{62}$-HA or mock transfected to control for nonspecific immunoprecipitaton. Two days post-transfection, cells were lysed, and a fraction of the cell lysates was immunopurified using a mouse monoclonal anti-HA antibody. Stau $1^{55}$-HA (lane 1), Stau $2^{59}$ HA (lane 2), and Stau2 ${ }^{62}$-HA (lane 3) were identified on Western blotting using a rabbit polyclonal anti-HA antibody. (Lane 4) IP of mock transfected cells. (B) Analysis of the RNA content of Staufencontaining mRNPs. From the immunoprecipitates described in $A$, RNA was extracted and run on a Bioanalyser RNA pico chip (Agilent) as indicated. Total RNA was also extracted from HEK293T cells. Migration profile of HEK293T cells' total RNA sample shows mainly two peaks corresponding to $18 \mathrm{~S}$ and $28 \mathrm{~S}$ ribosomal RNAs. Note that Bioanalyser profiles are not all plotted on the same absorbance scale.
Affymetrix. This experiment allowed us to perform a genome-wide identification of Stau1- and Stau2-associated mRNAs. To identify Staufen-HA-associated mRNAs, we performed a statistical (unpaired) analysis on mean-scale normalized MAS5 data with a $P$-value threshold of 0.05 . We then kept genes for which the hybridization signal was at least 2.5 times stronger with probes derived from Staufen-HA-expressing cells than with probes made from their respective controls. Similarly, total RNA from untransfected HEK293T cells was used to generate probes for microarray hybridization.

First, arrays hybridized with probes synthesized from HEK293T cell total RNA showed that 19,491 probe sets corresponding to 12,188 unique genes are expressed in these cells. This corresponds to $37.3 \%$ of the total probe sets on the microarray. Then, in order to identify Stau1 ${ }^{55}$ HA-associated mRNAs, four independent immunoprecipitations were performed from extracts of Stau ${ }^{55}$-HA transfected cells and mock transfected control cells. Hybridization results identified 1384 probe sets (7\% of expressed HEK293T mRNAs) that were enriched by more than 2.5-fold over control IP (Supplemental Table S1). Similarly, two independent hybridization experiments performed with mRNA isolated from Stau $2^{59}$-HA transfected cells and controls identified 2357 specific probe sets (12\% of expressed HEK293T mRNAs) (Supplemental Table S2). Using mRNA isolated from Stau2 ${ }^{62}$-HA transfected cells and controls, 2008 (10\% of expressed HEK293T mRNAs) probe sets were detected (Supplemental Table S3).

\section{A large percentage of $m R N A$ is specific for either Stau $1^{55}$ - or Stau2-associated mRNPs}

Then, the population of mRNAs associated with Stau1 ${ }^{55}$ HA mRNPs was compared with the mRNAs associated with Stau2-HA mRNPs (Fig. 3). Our data indicated that $63 \%$ and $58 \%$ of the probe sets identified with the Stau $1^{55}$ HA-derived probes were not found in $\mathrm{Stau}_{2}{ }^{62}$-HA or Stau $2^{59}$-HA mRNPs, respectively, and $49 \%$ are specific to Staul-containing mRNPs. The reverse is also true, since $75 \%$ and $74 \%$ of the probe sets identified with Stau2 ${ }^{59}$-HA- or Stau2 $2^{62}$-HA-associated mRNAs, respectively, were not found among the Stau $1^{55}$-associated mRNA. These results are consistent with the previous reports that Stau1 and Stau2 $2^{59}$ and Stau $2^{62}$ define different populations of RNA granules (Duchaine et al. 2002; Thomas et al. 2005). Nevertheless, 368 probe sets were common to all Staufen-containing mRNPs.

\section{Stau $2^{62}$ - and Stau2 ${ }^{59}$-associated mRNPs share common mRNAs}

Analyses of the Staufen2-associated mRNAs revealed that a greater proportion of probe sets are shared by the two Staufen2 isoforms as compared with what we described 


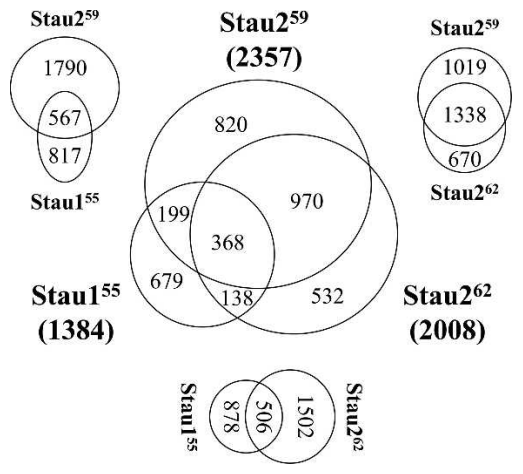

FIGURE 3. Venn diagrams summarizing microarray analysis results. The RNA content isolated from Stau1 ${ }^{55}$-HA, Stau $2^{59}-\mathrm{HA}$, and Stau $2^{62}$ HA IP (Fig. 2) was used to synthesize cRNA probes in order to hybridize Affymetrix HG_U133_plus_2 microarrays. For each hybridization, probe sets showing an enrichment of at least 2.5 -fold over the control IPs were identified. The numbers of probe sets that are either specific or common to Staufen paralogs are plotted in Venn diagrams.

above for Stau1 versus Stau2. Indeed, only $33 \%$ of the probe sets identified with probes derived from Stau $2^{62}$-HA mRNPs were uniquely found in these mRNPs, and $43 \%$ of the probe sets identified with probes derived from Stau $2^{59}$ HA mRNPs were specific for these mRNPs (Fig. 3). A total of 1338 probe sets were common to both Stau2-containing mRNPs. These results revealed that, although found in different mRNP complexes, these differentially spliced
Stau2 isoforms share several common mRNAs, suggesting that they play overlapping and/or complementary roles that finely regulate similar cellular pathways.

\section{RT-PCR validation of enriched mRNAs}

According to the microarray analyses, we chose several mRNAs identified as being specifically enriched in Stau $1^{55}$ HA, Stau $2^{59}$-HA, or Stau2 ${ }^{62}$-HA mRNPs to confirm their differential association with mRNPs (Table 1). First, cells were transfected with plasmids coding for either Stau $1^{55}$ HA, Stau $2^{62}$-HA, or Stau2 ${ }^{59}$-HA, and the resulting proteins were immunoprecipitated with anti-HA antibodies. Immunoprecipitates from mock transfected cells were used as control. Western blot showed that the HA-tagged proteins have been immunoprecipitated in equal amounts (Fig. 4A). Their expression was then compared with that of the endogenous proteins (Fig. 4B,C). Coimmunoprecipitated mRNAs were isolated and RT-PCR amplified with specific primers to validate the microarray results. As seen in Figure 5A, RNAs coding for FMN2 and IRF2BP2 were enriched in Stau $1^{55}$-HA-containing mRNPs, whereas RNAs coding for UQCRC2 and LTA4H and for MLLT11 and PPARGC1 were enriched in Stau2 $2^{62}$-HA- and Stau2 ${ }^{59}$-HA-containing mRNPs, respectively, as expected from the microarray analyses. Other mRNAs such as those coding for EIF5 and ZDHHC21 were common to Stau2 isoforms but not

TABLE 1. List of genes differentially found in Stau1 or Stau2 mRNPs and used for RT-PCR amplification

\begin{tabular}{|c|c|c|c|c|c|}
\hline \multirow[b]{2}{*}{ Gene name } & \multirow[b]{2}{*}{ Symbol } & \multirow[b]{2}{*}{ Probe set } & \multicolumn{3}{|c|}{$\begin{array}{l}\text { Fold increase in RNP as } \\
\text { compared with controls }\end{array}$} \\
\hline & & & Stau $1^{55}$ & Stau $2^{62}$ & Stau $2^{59}$ \\
\hline \multicolumn{6}{|l|}{ Probe sets enriched in Stau $1^{55} \mathrm{mRNPs}$} \\
\hline Formin 2 & FMN2 & 223618_at & 3.4 & 0.8 & 1.0 \\
\hline Interferon regulatory factor 2 binding protein 2 & IRF2BP2 & 224570_s_at & 4.2 & 0.9 & 1.1 \\
\hline Zinc finger protein 236 & ZNF236 & 222227_at & 17.7 & 0.3 & 0.6 \\
\hline \multicolumn{6}{|l|}{ Probe sets enriched in Stau $2^{62}$ mRNPs } \\
\hline Leukotriene A4 hydrolase & LTA4H & 208771_s_at & 1.1 & 2.7 & 0.8 \\
\hline Ubiquinol-cytochrome c reductase core protein II & UQCRC2 & 212600_s_at & 1.3 & 3.5 & 1.1 \\
\hline \multicolumn{6}{|l|}{ Probe sets enriched in Stau $2^{59}$ mRNPs } \\
\hline Myeloid/lymphoid or mixed-lineage leukemia; translocated 11 & MLLT11 & 211071_s_at & 2.1 & 2.1 & 5.5 \\
\hline Peroxisome proliferative activated receptor gamma coactivator 1 & PPARGC1 & 219195_at & 1.0 & 1.6 & 3.0 \\
\hline \multicolumn{6}{|l|}{ Probe sets enriched in both Stau $2^{59}$ and Stau $2^{62}$ mRNPs } \\
\hline Eukaryotic translation initiation factor $5 \mathrm{~A}$ & EIF5 & 208707_at & 1.3 & 4.9 & 6.8 \\
\hline Zinc finger DHHC domain containing 21 & ZDHHC21 & 243835_at & 0.7 & 3.7 & 5.7 \\
\hline Thioredoxin domain containing 9 & TXNDC9 & 203008_x_at & 1.3 & 7.0 & 4.0 \\
\hline \multicolumn{6}{|l|}{ Probe sets enriched in both $S t a u 1^{55}$ and Stau $2^{62}$ mRNPs } \\
\hline Acyl-Coenzyme A oxidase 1 palmitoyl & Acox1 & 227962_at & 8.7 & 8.1 & 6.7 \\
\hline $3^{\prime}\left(2^{\prime}\right) 5^{\prime}$-bisphosphate nucleotidase 1 & BPNT1 & 232103_at & 9.8 & 8.6 & 4.8 \\
\hline \multicolumn{6}{|l|}{ Control probe sets } \\
\hline Ubiquinol-cytochrome c reductase binding protein & UQCRB & 209065_at & 0.3 & 0.2 & 0.2 \\
\hline Likely ortholog of immediate early response erythropoietin 4 & LEREPO4 & 201595_s_at & 0.3 & 0.06 & 0.08 \\
\hline Growth and transformation-dependent protein & E2IG5 & 221533_at & 2.4 & 2.1 & 1.3 \\
\hline
\end{tabular}


A

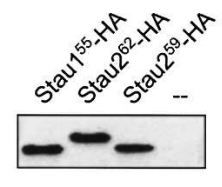

WB : $\alpha-H A$
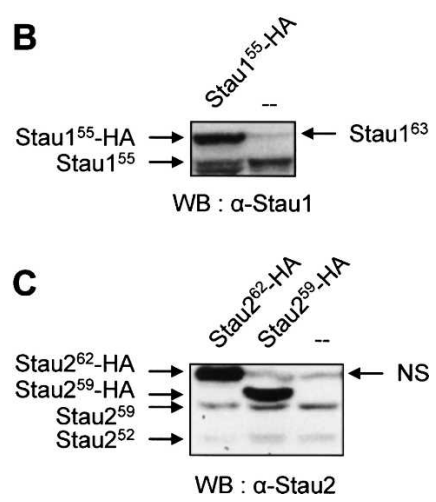

FIGURE 4. Expression of Staufen-tagged proteins. HEK293T cells were transfected with plasmids coding for either Stau $1^{55}-\mathrm{HA}$, Stau $2^{62}$ HA, or Stau $2^{59}$-HA. Mock-transfected cells were used as controls. Forty-eight hours post-transfection, cells were lysed, and Staufencontaining mRNPs were immunoprecipitated using anti-HA antibodies. Proteins in the immunoprecipitates were analyzed by Western blot analysis using anti $(\alpha)$-HA $(A)$, anti-Stau1 $(B)$, or anti-Stau2 $(C)$ antibodies. (NS) Nonspecific band.

Staul mRNPs. Some of the precipitated mRNAs expected to be unique to one mRNP according to microarray analyses were nevertheless slightly enriched in the other mRNPs as compared with controls. It is possible that the overexpression of the tagged proteins has facilitated the formation of a relatively low number of heterogeneous granules with the endogenous proteins. Finally, mRNAs coding for BPNT1 and Acoxl that were enriched in each mRNP according to the microarray data are also common to mRNPs in the RT-PCR asssay. RT-PCR amplification of serial dilutions of mRNAs commonly enriched in the three mRNPs indicated that the amplifications were in the linear range (Fig. 5B,C). Consistent with the microarray data, these results show that specific mRNA can be differentially enriched in different mRNPs, whereas others are common to both isoforms.

Then, we tested whether transcripts are also enriched in endogenous Stau1- and Stau2-containing mRNPs. Endogenous Stau1- and Stau2-containing mRNPs were immunopurified using anti-Staul and anti-Stau 2 antibodies, respectively. Nonimmune sera were used as controls. Coimmunoprecipitated mRNAs were isolated and RTPCR amplified with specific primers. Consistent with the microarray data, mRNAs coding for IRF2BP2, FMN2, and ZNF236 were enriched in the Staul mRNP extracts as compared with their respective controls (Fig. 6A). Except for IRF2BP2 mRNA, they were not enriched in Stau2containing mRNPs (Fig. 6B). Similarly, mRNAs coding for
EIF5, LTA4H, and TXNDC9 were enriched in Stau2containing mRNPs as compared with controls (Fig. 6B). However, an enrichment was also seen in Staul immunoprecipitates as compared with controls (Fig. 6A), suggesting either that the anti-Staul antibody is not as specific as observed by Western blotting or that the endogenous Stau1containing granules are more heterogeneous than expected. If so, the number of mRNAs common to Stau1- and Stau2containing mRNPs may well be slightly higher than that seen by microarrays. Other mRNAs that were not enriched

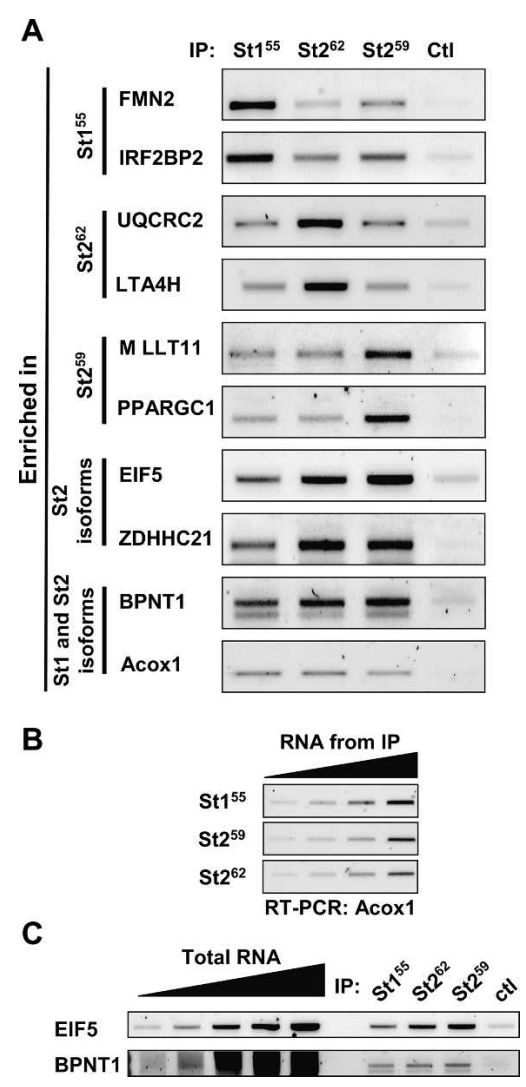

FIGURE 5. RT-PCR amplification of selected mRNA following mRNPs immunoprecipitation. HEK293T cells were transfected with plasmids coding for either Stau $1^{55}$-HA $\left(\mathrm{St}_{1}{ }^{55}\right)$, Stau $2^{62}-\mathrm{HA}\left(\mathrm{St}_{2}{ }^{62}\right)$, or Stau $2^{59}$-HA $\left(\mathrm{St}^{29}\right)$. Mock transfected cells were used as controls (Ctl). Forty-eight hours post-transfection, cells were lysed, and Staufen-containing mRNPs were immunoprecipitated (IP) using anti-HA antibodies. Co-immunoprecipitated mRNAs were purified and RT-PCR amplified with pairs of oligonucleotides specific for the indicated genes (Supplemental Table S7). (A) Genes were chosen according to their differential enrichment in Stau $1^{55}$-HA, Stau2 ${ }^{59}$-HA, and Stau $2^{62}$-HA mRNPs according to the microarray data. Serial dilutions of mRNA in the immunoprecipitates $(B)$ or in cell extracts (C) were used to indicate that RT-PCR amplifications were in the linear range. (FMN2) forming 2, (IRF2BP2) interferon regulatory factor 2 binding protein 2, (LTA4H) leukotriene A4 hydrolase, (EIF5) eukaryotic translation initiation factor 5A, (Acox1) acyl-Coenzyme A oxidase 1 palmitoyl, (BPNT1) 3' 3 $^{\prime}$ ) 5' -bisphosphate nucleotidase 1, (MLLT11) ALL1-fused gene from chromosome 1q, (PPARGC1) peroxisome proliferative activated receptor gamma coactivator 1 , (UQCRC2) ubiquinol-cytochrome c reductase core protein II, (ZDHHC21) zinc finger DHHC domain containing 21. 
A

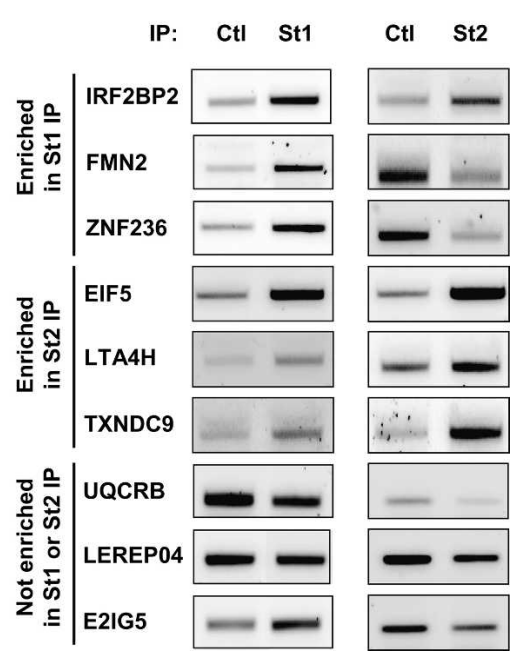

FIGURE 6. RT-PCR amplification of selected endogenous mRNA following Stau1 or Stau2 immunoprecipitation. HEK293T cells were lysed, and Stau1- $(A)$ or Stau2-containing $(B)$ mRNPs were immunoprecipitated (IP) with mouse monoclonal anti-Stau1 (St1) or rabbit polyclonal anti-Stau2 (St2) antibodies, respectively. Immunoprecipitation with preimmune sera was used as controls (Ctl). Coimmunoprecipitated mRNAs were isolated and RT-PCR amplified with pairs of oligonucleotides specific for the indicated genes (Supplemental Table S7). (IRF2BP2) interferon regulatory factor 2 binding protein 2 , (FMN2) formin 2, (ZNF236) zinc finger protein 236, (EIF5) eukaryotic translation initiation factor 5A, (LTA4H) leukotriene A4 hydrolase, (TXNDC9) thioredoxin domain containing 9, (UQCRB) ubiquinol-cytochrome $\mathrm{c}$ reductase binding protein, (LEREPO4) likely ortholog of mouse immediate early response erythropoietin 4, (E2IG5) growth and transformation-dependent protein.

in the mRNPs over the controls in the microarray analyses also showed no enrichment by RT-PCR analysis, being found in both mRNP and controls. Altogether, these results confirm that specific mRNAs are preferentially found in Stau1- or Stau2-containing mRNPs as revealed by the microarray analyses, but the number of common mRNAs may be slightly underestimated.

\section{Enriched mRNAs are involved in diverse cellular functions}

We next tried to identify pathways in which Staul and Stau2 are involved as a means to understanding the biological functions of Staufen. Probe set lists have been analyzed with the DAVID functional annotation tool (Dennis et al. 2003) to assign cellular functions to mRNAs that were enriched in Staufen-containing RNPs. The result from GO classification reveals that mRNAs associated with Stau1- and Stau2-containing RNPs are distributed roughly in the same functional and molecular categories (Table 2; Supplemental Table S4). These include mRNAs coding for genes involved in cellular metabolism and cellular physiological processes, localization and transport, transcription, alternative splicing, metal-ion binding, and catalytic activ- ities. Similarly, the subcellular distribution of the proteins encoded by the probe sets is quite similar between Stauland Stau2-associated mRNAs. Probe sets were then analyzed with the Babelomics software (Al-Shahrour et al. 2005) to identify common probe sets in each GO category. Interestingly, despite the fact that Stau1- and Stau2-derived probe sets are distributed essentially in the same functional categories, the nature of the genes encoded by these probe sets is quite different in each category (Supplemental Table S5), only $\sim 30 \%$ of them being common. These results suggest that Stau1 and Stau2 play complementary roles that nevertheless fulfill similar functions.

GO classification was also done with mRNAs associated with Stau $2^{62}$ and Stau $2^{59}$ mRNPs (Table 2; Supplemental Table S4). RNAs associated with these mRNPs are linked to the same GO categories. In addition, in each category, about $60 \%$ of the genes are common (Supplemental Table S6), suggesting that they play redundant functions.

\section{DISCUSSION}

\section{Staufen-bound mRNAs}

Staufen proteins are RNA-binding proteins ubiquitously expressed in animals that play several roles in mRNA transport, translation, and mRNA decay. Therefore, they are likely multifunctional proteins involved in several pathways and consequently should bind mRNAs that are not necessarily functionally related. In this paper, a genome-wide approach was used to identify mRNAs that are associated with either Stau1- or Stau2-containing particles. Independent RT-PCR analyses of several putative mRNA targets confirmed the reliability of this technique. Our results further indicate that these mRNAs are components of endogenous Stau1- and/or Stau2-containing mRNPs. The gene ontology distribution of these mRNAs allowed us to unveil the major pathways in which Stau1 and Stau2 proteins are involved. Our results with the microarray screens indicate that Staul and Stau2 associate with different but overlapping populations of mRNAs and that these mRNAs are nevertheless involved in common pathways. It is consistent with the high degree of sequence similarity between Stau1 and Stau2 that predicts that they may have conserved convergent functions and with the observation that they are distributed in distinct mRNP complexes in neurites. However, the more sensitive RTPCR experiment suggests that the affinity of specific mRNAs for Stau1- or Stau2-containing mRNPs may not be all or none. Indeed, following overexpression of tagged proteins, mRNAs are enriched in specific mRNPs as predicted by the microarray analyses, but most of them are also enriched in the other IPs relative to the control, although to a lesser degree of enrichment. The same trend is observed with the endogenous mRNPs, especially in Stau1-containing complexes. Although it could be due to 
TABLE 2. Summary of DAVID GO classification of target mRNA population

\begin{tabular}{|c|c|c|c|c|}
\hline Gene ontology group & $\begin{array}{c}\text { HEK293 } \\
(\%)\end{array}$ & $\begin{array}{c}\text { Stau1 }{ }^{55} \\
(\%)\end{array}$ & $\begin{array}{c}\text { Stau2 } 2^{59} \\
(\%)\end{array}$ & $\begin{array}{c}\text { Stau2 }{ }^{62} \\
(\%)\end{array}$ \\
\hline Activator & 1.95 & 2.20 & 2.2 & 2.3 \\
\hline Alternative splicing & 18.52 & 18.04 & 18.0 & 18.2 \\
\hline Catalytic activity & 25.77 & 22.44 & 24.6 & 25.4 \\
\hline Cellular metabolism & 36.88 & 30.02 & 34.0 & 34.3 \\
\hline Cellular physiological process & 48.72 & 41.70 & 46.8 & 46.6 \\
\hline Cellular protein metabolism & 15.02 & 12.74 & 14.5 & 15.4 \\
\hline Establishment of protein localization & 3.72 & 3.26 & 4.1 & 3.4 \\
\hline Glycoprotein metabolism & & 1.06 & 1.0 & 1.0 \\
\hline Ligase activity & 2.98 & 3.03 & 3.4 & 4.1 \\
\hline Ligase activity, forming carbon-nitrogen bonds & 2.21 & 2.58 & 2.9 & 3.5 \\
\hline Macromolecule metabolism & 22.95 & 18.50 & 20.3 & 21.4 \\
\hline Magnesium ion binding & 1.67 & 1.90 & 2.1 & 2.2 \\
\hline Metabolism & 39.12 & 32.15 & 36.0 & 36.2 \\
\hline Metal binding & 11.62 & 11.37 & 11.8 & 12.3 \\
\hline Mutagenesis site & 5.45 & 5.84 & 5.8 & 6.0 \\
\hline Nucleic acid metabolism & 17.99 & 14.18 & 15.8 & 15.5 \\
\hline Physiological process & 51.69 & 44.20 & 49.4 & 49.1 \\
\hline Primary metabolism & 35.98 & 29.64 & 33.6 & 33.6 \\
\hline Protein catabolism & 1.26 & 1.29 & 1.7 & 1.8 \\
\hline Protein degradation & 0.20 & 0.53 & 0.4 & 0.4 \\
\hline Protein localization & 3.81 & 3.34 & 4.2 & 3.5 \\
\hline Protein metabolism & 16.33 & 13.80 & 16.1 & 16.8 \\
\hline Protein modification & 8.63 & 8.26 & 10.2 & 10.7 \\
\hline Protein transport & 3.63 & 3.11 & 4.1 & 2.4 \\
\hline Protein ubiquitination & 1.39 & 1.67 & 1.8 & 2.3 \\
\hline Regulation of cellular metabolism & 11.94 & 10.46 & 11.2 & 11.2 \\
\hline Regulation of cellular physiological process & 16.15 & 14.10 & 15.3 & 14.7 \\
\hline Regulation of metabolism & 12.28 & 10.46 & 11.4 & 11.4 \\
\hline Regulation of nucleic acid metabolism & 11.27 & 9.86 & 10.8 & 10.3 \\
\hline Regulation of transcription & 11.06 & 9.70 & 10.6 & 10.1 \\
\hline Splice variant & 18.42 & 18.35 & 18.0 & 18.2 \\
\hline Transcription & 11.66 & 10.24 & 11.4 & 10.9 \\
\hline Transferase activity & 9.00 & 8.34 & 9.1 & 8.4 \\
\hline Transition metal-ion binding & 12.30 & 11.90 & 12.3 & 12.2 \\
\hline Ubiquitin cycle & 3.14 & 3.11 & 4.2 & 4.7 \\
\hline Ubiquitin ligase complex & 1.45 & 1.67 & 2.0 & 2.4 \\
\hline Ubiquitin-protein ligase activity & 1.90 & 2.05 & 2.6 & 3.3 \\
\hline Ubl conjugation pathway & 0.42 & 0.61 & 2.8 & 2.9 \\
\hline Vesicle-mediated transport & 2.11 & 1.97 & 2.6 & 2.5 \\
\hline Zinc ion binding & 10.52 & 10.01 & 10.4 & 10.6 \\
\hline Zinc finger & 8.09 & 8.04 & 8.4 & 8.9 \\
\hline Cytoplasm & 18.91 & 15.69 & 18.1 & 18.6 \\
\hline Endoplasmic reticulum & 2.32 & 3.11 & 3.4 & 3.7 \\
\hline Golgi apparatus & 2.78 & 3.71 & 3.7 & 3.5 \\
\hline Intracellular membrane-bound organelle & 31.28 & 25.85 & 29.0 & 29.3 \\
\hline Intracellular organelle & 35.15 & 28.28 & 31.6 & 32.0 \\
\hline Nuclear protein & 18.26 & 14.56 & 15.8 & 15.9 \\
\hline Nucleus & 21.57 & 17.21 & 18.8 & 19.1 \\
\hline Organelle & 35.16 & 28.28 & 31.6 & 32.0 \\
\hline Organelle membrane & 2.64 & 2.43 & 2.6 & 2.6 \\
\hline
\end{tabular}

regulation of the post-transcriptional expression of these mRNAs according to cell needs. Alternatively, it is possible that a certain percentage of heterogeneous granules may form in these cells, causing the immunoprecipitation of both paralogs in different proportions.

This study also allows us to identify the level of divergence in the RNA content of two mRNPs that contain protein isoforms with identical RNAbinding domains. Stau2 ${ }^{62}$ and Stau2 ${ }^{59}$ are differentially spliced isoforms that only differ at their N-terminal extremity. In neurons, they form distinct mRNPs that fractionate with ribosome-free RNPs and with ribosomes, respectively (Duchaine et al. 2002). Our results indicate that these mRNPs contain a high percentage of shared mRNAs, suggesting that these isoforms play redundant functions. However, about one-third of the associated mRNAs are unique to either Stau $2^{62}$ or Stau $2^{59}$ mRNPs, indicating that the Stau2 RNA-binding domain is not the sole determinant for the selection of associated mRNAs. It is consistent with proteomic analyses that showed that RNA granules contain several RNAbinding proteins that may, in theory, bind specific mRNAs. RNA granules are likely heterogeneous particles composed of several RNA-binding proteins and their associated RNAs. Stau2 was indeed found in several RNA granules along with other RNA-binding proteins such as pur proteins, DeadBox helicases, FMRP, and hnRNPs (Ohashi et al. 2002; Elvira et al. 2006b). All of them may bind RNA and associate with Staufen to form RNA granules. Alternatively, the accessibility of specific RNAs and/or the composition of specific RNPs may be dictated by the availability of mRNA subpopulations in subcellular compartments allowing specific association with differentially distributed Stau2 isoforms. technical limitations (overexpression, specificity of antibodies, variation in the amounts of mRNA in the controls), the sequence similarity between Stau1 and Stau2 may also explain the fact that mRNA can bind to the two Staufen paralogs with differential affinity. It may allow more precise

\section{GO distribution}

RNAs associated with Stau1- and Stau2-containing mRNPs essentially fall into the same GO categories, suggesting that these paralogs have overlapping and/or complementary 
functions. Although broadly distributed in several GO categories, these mRNAs code for proteins mainly involved in cell metabolism, cellular physiological processes, localization and transport, transcription, alternative splicing, metal-ion binding, and catalytic activities. These are cellular processes that need fine regulation to appropriately respond to cell needs and cell stimuli. Staufen-dependent post-transcriptional regulation of gene expression of a specific subpopulation of messengers would allow a cell to specifically and quickly respond to cell stimuli. Posttranslational modifications of mRNP-associated proteins, such as phosphorylation, may transduce the external stimuli into functional responses. Indeed, phosphorylation of the zipcode-binding protein $\mathrm{ZBP} 1$ by the protein kinase Src results in the dissociation of repressed mRNAs from mRNPs and in their translation on polyribosomes (Huttelmaier et al. 2005).

Of interest is also the association between Staul- and Stau2-bound mRNAs and localization and transport. It is well documented that one of the functions of both Stau1 and Stau2 is related to mRNA transport and localization. In polarized cells such as neurons, Stau1 and Stau2 are found in the form of RNA granules that move in dendrites (Kiebler et al. 1999; Kohrmann et al. 1999; Tang et al. 2001; Duchaine et al. 2002; Barbee et al. 2006; Kim and Kim 2006). Down-regulation of Staul using RNAi or impaired transport of Stau2 following expression of a dominant negative mutant both reduce the transport of mRNA in dendrites. Our results now suggest that Stau1 and Stau 2 may also regulate their own function by controlling the post-transcriptional expression of proteins involved in the mechanics of transport and localization. Similarly, several mRNAs are linked to the ubiquitination process. To finely regulate signals induced by cell stimulation, it is important to quickly inactivate and/or eliminate signaling proteins. One efficient pathway relies on protein degradation by the proteasome, a process that is dependent on the ubiquitination of proteins to be degraded (Ciechanover et al. 2000). It is likely that mRNA transport and local translation is balanced by local protein degradation, allowing fine regulation of cell signaling. Components of the proteasome are found in dendrites, and the function of the proteasome is necessary for long-term potentiation (Hegde et al. 1997; Fonseca et al. 2006; Karpova et al. 2006).

\section{Comparison to other RNPs}

Our data indicate that there is an overlap between Stau1and Stau2-associated mRNAs. We decided to look if there is also an overlap with other known mRNA populations associated with other RNA-binding proteins. FMRP is the product of the Fmr1 gene. Absence or mutation of FMRP causes Fragile X syndrome, an X-linked form of mental retardation. It has been shown that FMRP is involved in translational repression and in synaptic plasticity (Zalfa et al.
2006). It was reported that FMRP copurifies with at least 432 different mRNAs following immunoprecipitation from mouse brain extracts (Brown et al. 2001). FRMP is also found in complex with Staul (Brendel et al. 2004; Villace et al. 2004), which suggests that there could be a common set of target mRNAs for both proteins. In order to test this possibility, we wanted to compare the 432 mRNAs previously identified as potential FMRP targets with the Stau $1^{55}$-HA-associated mRNA population identified in the present study. Unfortunately, it is hard to gain a global picture of the relationships that exist between the RNA content of different mRNPs that were characterized using the RIP-chip approach. Comparisons are difficult to make between datasets available in the literature because of high variations in (1) the array platforms that are used; (2) the tissue or cell type used as starting material; (3) the developmental stage at which the experiment was conducted; and (4) the species that were used to study mRNA content of mRNPs. Nevertheless, we first performed a search for probe sets corresponding to the human orthologs of mouse probe sets. From the 432 probe sets corresponding to FMRP-associated mRNAs, 375 probe sets possess a human ortholog. These 375 probe sets represent 355 unique genes. Human U133 gene chip contains 1363 probe sets that represent the orthologs of the 375 mouse probe sets. Of these 1363 probe sets, $58(4.3 \%)$ are enriched in Stau $1^{55}$ HA purification. These 58 probe sets represent 52 unique genes, which leads to the conclusion that $\sim 15 \%(52 / 355)$ of FMRP-associated mRNAs are also enriched in Stau1 ${ }^{55}$-HA purification (Table 3). Similarly, the population of mRNAs associated with Staul is distinct from that of mRNA bound to the human zipcode binding protein IMP. Indeed, only 26 probe sets (Table 4 ) are common among the 352 (7\%) probe sets in IMP1 complexes and Stau1 (1.6\%) complexes. These two RNA granules do not colocalize in HEK293T cells (Jonson et al. 2007).

\section{Staufen-binding site}

To understand the precise role of Staufen in mammalian cells, it will be necessary to determine how Staufen regulates the fate of each of its associated mRNAs and therefore how it regulates the function of the encoded protein. First, it must be determined whether Staul or Stau2 directly bind RNP-associated RNAs or whether these RNA copurify by virtue of their binding to other RNAbinding proteins in the complexes. This is not a trivial task, since Stau1 binds any structured RNA in vitro (Ramos et al. 1999, 2000; Wickham et al. 1999). Second, the RNA structure of the Stau1- and Stau2-binding sites and their position relative to the $5^{\prime}$ or $3^{\prime}$ UTR of each mRNA must be determined. The position of the Stau1-binding site in a particular mRNA is of primary importance since it dictates whether the mRNA is targeted to polysomes for enhanced translation (Dugre-Brisson et al. 2005) or is degraded by 
TABLE 3. mRNAs associated with both Stau1 ${ }^{55}$-HA (this study) and FMRP (Brown et al. 2001)

\begin{tabular}{|c|c|c|}
\hline Stau $1^{55}$-HA/FMRP-associated mRNAs & HG_U133 & MG_U74v2 \\
\hline Adenylate cyclase 1 (brain) & 213245_at & 16691_AT \\
\hline AP1 gamma subunit binding protein 1 & 239678_at & 133560_AT \\
\hline AT rich interactive domain 2 (ARID, RFX-like) & 225486_at & 103079_AT \\
\hline ATM/ATR-substrate Chk2-interacting $\mathrm{Zn}^{2+}$ finger protein & 201854_s_at & 130730_F_AT \\
\hline ATPase, Class VI, type $11 \mathrm{~A}$ & 230875_s_at & 103899_AT \\
\hline Cdk5 and Abl enzyme substrate 1 & 225531_at & 117085_AT \\
\hline Chromosome 15 open reading frame 39 & 215087_at & 104389_AT \\
\hline Chromosome 6 open reading frame 107 & 226135_at & 103773_AT \\
\hline Chromosome 9 open reading frame 10 & 15555908_at & 91488_ĀT \\
\hline Chrondoitin polymerizing factor & 202175_at & 97387_AT \\
\hline DIP2 disco-interacting protein 2 homolog A (Drosophila) & 1552677_a_at & 114468_AT \\
\hline Dipeptidyl-peptidase 9 & 230018_at & 114469_AT \\
\hline Dymeclin & 223171_at & 99988_AT \\
\hline Eukaryotic translation initiation factor 4 gamma, 3 & 243149_at & 96850_AT \\
\hline Exostoses (multiple)-like 1 & 206329_at & 115969_AT \\
\hline Glucocorticoid receptor DNA binding factor 1 & 229394_s_at & 96208_AT \\
\hline Guanine nucleotide binding protein (G protein) alpha 1 & 221737_at & 97226_AT \\
\hline Hypothetical protein DKFZp76112123 & 54970_at & 102271_AT \\
\hline Jun D proto-oncogene & 203752_s_at & 1022364_AT \\
\hline Kelch-like 21 (Drosophila) & 203068_at & 104293_AT \\
\hline KIAA0226 & 212735_at & 111540_AT \\
\hline KIAA0284 & 213242_x_at & 131216_F_AT \\
\hline KIAA0317 & 202128_at & 109944_AT \\
\hline KIAA0515 & 212069_s_at & 107033_AT \\
\hline Latrophilin 1 & 219145_at & 112497_AT \\
\hline Leucine-rich repeat neuronal $6 \mathrm{~A}$ & 227933_at & 139527_AT \\
\hline MCM3 minichromosome maintenance deficient 3 & 212269_s_at & 97433_ĀT \\
\hline Meningioma (disrupted in balanced translocation) 1 & 205330_at & 110774_AT \\
\hline Nischarin & 227392_at & 94236_AT \\
\hline Nuclear factor I/C (CCAAT-binding transcription factor) & 226895_at & 92241_AT \\
\hline $\mathrm{PH}$ domain and leucine rich repeat protein phosphatase & 212719_at & 109399_AT \\
\hline Phosphatidylinositil 4-kinase, catalytic, alpha polypeptide & 207081_s_at & 104208_AT \\
\hline Protein phosphatase 1 , regulatory subunit $9 \mathrm{~B}$ & 225124_at & 112345_AT \\
\hline PRP8 pre-mRNA processing factor 8 homolog & 200000_s_at & 98574_AT \\
\hline RAB6 interacting protein 1 & 212561_at & 104108_AT \\
\hline Ral quanine nucleotide dissociation stimulator & 20050_at & 100530_AT \\
\hline SET domain containing $1 \mathrm{~B}$ & 213153_at & 129315_AT \\
\hline Spectrin domain with coiled-coils 1 & 240765_at & 102942_AT \\
\hline Steril alpha motif domain containing $4 \mathrm{~B}$ & 227511_at & 100889_AT \\
\hline Synaptotagmin IX & 1563658_a_at & 107392_AT \\
\hline TBC1 domain family, member 16 & 228488_at & 110258_AT \\
\hline TBC1 domain family, member 24 & 227908_at & 97710_F_AT \\
\hline Transmembrane protein 1 & 1555446_s_at & 104202_AT \\
\hline Tyrosine kinase, nonreceptor, 2 & 2033839_s_at & 102850_AT \\
\hline Ubiquitin protein ligase E3 component n-recognin 2 & 212760_at & 111342_AT \\
\hline Ubiquitin-specific peptidase 2 & 229337_at & 92820_ĀT \\
\hline Unc-51-like kinase 1 (Caenorhabditis elegans) & 209333_at & 102332_AT \\
\hline WW domain containing E3 ubiquitin protein ligase 2 & 204022_at & 103717_AT \\
\hline WW, $\mathrm{C} 2$, and coiled-coil domain containing 1 & 241950_at & 96518_AT \\
\hline Zinc finger protein 236 & 222227_at & 115194_AT \\
\hline Zinc finger protein 42 (Myeloid-specific retinoic acid receptor) & 40569_at & 94101_ĀT \\
\hline ZXD family zinc finger $\mathrm{C}$ & 230209_at & 117039_AT \\
\hline
\end{tabular}

the Staul-mediated mRNA decay pathway (Kim et al. 2005). Although a gene-by-gene approach is required at least at the beginning to characterize the Staufen1-binding site, an in silico high-throughput technique to assess where Staul binds to a given group of mRNAs would facilitate the search. The classification presented here of Stau1- and Stau2-associated mRNAs in functional subclasses will be helpful in orienting further studies. We recently reported that Staul binds to a stem of 19 nucleotides (nt) in the $3^{\prime}$ UTR of the Arf1 mRNA (Kim et al. 2007). However, the 
TABLE 4. mRNAs associated with both Stau $1^{55}$-HA (this study) and IMP (Jonson et al. 2007)

\begin{tabular}{|c|c|}
\hline Stau $1^{55}$-HA/IMP-associated mRNAs & HG_U133 \\
\hline - & 224752_at \\
\hline $\begin{array}{l}\text { Amyotrophic lateral sclerosis } 2 \text { (juvenile) chromosome region, } \\
\text { candidate } 2\end{array}$ & 223266_at \\
\hline ASF1 anti-silencing function 1 homolog B (S. cerevisiae) & 218115_at \\
\hline Chromosome 1 open reading frame 160 & 52078_at \\
\hline Chromosome 22 open reading frame 16 & 224932_at \\
\hline Coagulation factor VIII-associated (intronic transcript) 1 & 203274_at \\
\hline Dymeclin & 223171_at \\
\hline Family with sequence similarity 89 , member B & 32209_at \\
\hline Gem (nuclear organelle) associated protein 7 & 222821_s_at \\
\hline Glucosamine-6-phosphate deaminase 1 & 202382_s_at \\
\hline Golgi reassembly stacking protein 2, $55 \mathrm{kDa}$ & 208842_s_at \\
\hline Hypothetical gene LOC283846 & 217948_at \\
\hline Leucine zipper and CTNNBIP1 domain containing & 212281_s_at \\
\hline Opioid receptor, sigma 1 & 201692_at \\
\hline OTU domain, ubiquitin aldehyde binding 1 & 38710_at \\
\hline Phosphatidylinositol glycan anchor biosynthesis, class S & 223148_at \\
\hline Phosphoribosylaminoimidazole carboxylase & 201013_s_at \\
\hline PRA1 domain family, member 2 & 203456_at \\
\hline Progestagen-associated endometrial protein & 227511_at \\
\hline Protein phosphatase 1, regulatory (inhibitor) subunit 11 & 1566303_s_at \\
\hline Pterin-4 alpha-carbinolamine dehydratase & 203557_s_at \\
\hline Rhomboid domain containing 2 & 222995_s_at \\
\hline Solute carrier family 38, member 6 & 214830_at \\
\hline Splicing factor $3 \mathrm{~b}$, subunit $4,49 \mathrm{kDa}$ & 209044_x_at \\
\hline TBC1 domain family, member 22B & 223375_at \\
\hline Zinc finger protein 236 & 222227_at \\
\hline
\end{tabular}

four hybridizations. This is also true for all other previously identified probe sets that are either enriched in the four new microarrays but were not retained in this study because of their $P$-values above the threshold (three probe sets), or are enriched in two (five probe sets) or three (five probe sets) of the four new hybridizations. Therefore, although not statistically significant, these probe sets are likely targets for Staul. Indeed, if microarray data are analyzed using the robust multiarray average (RMA) data processing method, 19 out of the 23 previously identified mRNAs (including Arf1) are identified. These data suggest that more mRNAs than those in the list may be associated with Staul.

We have for the first time showed, by an unbiased approach, that Staul and Stau2 associate with different although overlapping subsets of mRNAs in the cells. Interestingly, mRNAs associated with Stau1- and Stau2-containing mRNPs code for proteins that are sorted in similar GO categories, suggesting that the two paralogs play complementary roles in mammalian cells. It is possible that during evolution the Stau critical features that confer specific binding for Staul are still unclear, and adjacent uncharacterized region(s) are also needed for optimal binding. Indeed, in silico analyses of several Staul target mRNAs failed to identify a 19-nt stem in these mRNAs, indicating that the recognition element is probably more complex than a single stem. This is consistent with the Staufen-binding site in the Drosophila bicoid mRNA that included three stem-loops (Ferrandon et al. 1994). Similarly, other dsRNA-binding proteins were shown to bind complex RNA structures composed of stems, bulges, and loops (Herbert and Rich 2001; Hallegger et al. 2006). The nature of the Stau2-binding site is completely unknown.

We previously reported a partial list of Staul-associated mRNAs using the 22,000 probe sets microarrays from Affymetrix (Kim et al. 2005). We now extend this list with four additional independent hybridizations of the 54,000 probe sets microarrays. Among the 23 previously identified Staul-associated mRNAs, nine are present in the new Stau ${ }^{55}$-HA analysis. Notably, the probe set corresponding to Arf1 is not present in the list. However, Arf1 mRNA has been shown to be a real target of Staul (Kim et al. 2005), and its Stau1-binding site in the 3' UTR has been identified (Kim et al. 2007). Careful analysis of the novel hybridizations indicates that the probe set corresponding to Arf1 is enriched at least 2.5-fold over the controls in three of the gene has duplicated to finely regulate specific pathways. Differential splicing events in each gene then allow a further diversification of the repertoires of possible functions. Altogether our results are consistent with the post-transcriptional operon hypothesis (Keene and Tenenbaum 2002) for concerted expression of functionally related genes in specific pathways.

\section{MATERIALS AND METHODS}

\section{Immunopurifications}

HEK293T cells were propagated in DMEM medium supplemented with $10 \%$ cosmic calf serum (HyClone); $3 \times 10^{6}$ cells were plated in 150-mm Petri dishes, and transfections were performed $24 \mathrm{~h}$ after plating. Cells were transfected with one of the following plasmids: Stau1 ${ }^{55}-\mathrm{HA}_{3}$ (Duchaine et al. 2000), Stau $2^{59}-\mathrm{HA}_{3}$, or $\mathrm{Stau}_{2}{ }^{62}-\mathrm{HA}_{3}$ (Elvira et al. 2006a) using the calcium-phosphate coprecipitate technique. Mock cells transfected with untagged expressing vectors or the vector alone were used for control. More than $80 \%$ of the cells were transfected. Cells were collected and lysed $48 \mathrm{~h}$ after transfection. Immunopurification, using anti-HA antibody, was performed as previously described (Duchaine et al. 2000) except that Escherichia coli tRNA (Sigma-Aldrich) was added to a final concentration of $200 \mu \mathrm{g} / \mathrm{mL}$ during the incubation with Protein-A-Sepharose (GE healthcare). After extensive washing, the resin was incubated $5 \mathrm{~min}$ at $95^{\circ} \mathrm{C}$ in 
$1 \times$ Laemmli buffer. One-tenth of the eluate was kept for Western blot analysis; the remainder of the sample was used for RNA extraction. A rabbit polyclonal anti-HA antibody (Sigma) and Western blotting were used to detect transfected Stau1-HA and Stau2-HA proteins.

\section{Microarray analysis}

RNA was extracted with Trizol reagent (Invitrogen). The RNA pellet was solubilized in water, and residual contaminants were removed using an RNA nanoprep column (Stratagene). Biotinylated cRNA probes were synthesized by the two-cycle target labeling assay following the Affymetrix procedure. Affymetrix human genome arrays (HG-U133_Plus_2: comprising 52,245 probe sets that correspond to 29,555 unique genes) were used for hybridization according to Affymetrix guidelines. Hybridized chips were scanned using an Affymetrix GeneChip 3000 Scanner. Results were recorded using the GeneChip Operating Software (GCOS) platform, which includes the GeneChip Scanner 3000 high-resolution scanning patch that enables feature extraction (Affymetrix). Notably, the Affymetrix Gene Expression Assay identifies changes that are greater than twofold with $98 \%$ accuracy (Wodicka et al. 1997).

To identify mRNAs that specifically copurify with Stau $1^{55}$-HA, Stau $2^{59}-\mathrm{HA}$, and Stau2 ${ }^{62}-\mathrm{HA}$, signal intensities obtained for specific IPs were compared with the baseline signal intensities of control IPs using statistical analysis on mean-scale normalized MAS5 data with a $P$-value limit of 0.05 . The same methodology was used to compare microarrays hybridized with cRNAs synthesized from specific IPs to cRNAs synthesized from the total RNA content of HEK293T cells. Each probe set presenting a fold enrichment over control of more than 2.5 was scored as a potential Staufen-associated mRNA. Only the probe sets showing a difference of more than 200 arbitrary units of intensity between the IP and control were kept for further analysis. Complete microarray analysis results can be found in the Supplemental Data. Microarray data have been deposited in the GEO database and are available through the series accession numbers GSE8437 and GSE8438.

\section{RT-PCR amplification}

RT-PCR was performed as previously described (Kim et al. 2005). Primer pairs are listed in Supplemental Table S7.

\section{SUPPLEMENTAL DATA}

Supplemental material can be found online at www.rnajournal.org.

\section{ACKNOWLEDGMENTS}

We thank the Genome Quebec Innovation Centre, in particular André Ponton, for microarray screening and analysis. We also thank Kirill Tarassov for comments on the manuscript. This work was supported by grants from the Natural Sciences and Engineering Research Council of Canada (NSERC) and Canadian Institutes of Health Research (CIHR) to L.D.G. L.F. and M.M.-L. were supported by scholarships from the Fonds de la recherche en santé du Québec (FRSQ) and the Natural Sciences and Engineering Research Council of Canada (NSERC), respectively.

Received July 6, 2007; accepted November 5, 2007.

\section{REFERENCES}

Al-Shahrour, F., Minguez, P., Vaquerizas, J.M., Conde, L., and Dopazo, J. 2005. BABELOMICS: A suite of web tools for functional annotation and analysis of groups of genes in highthroughput experiments. Nucleic Acids Res. 33: W460-W464. doi: 10.1093/nar/gki456.

Barbee, S.A., Estes, P.S., Cziko, A.M., Hillebrand, J., Luedeman, R.A., Coller, J.M., Johnson, N., Howlett, I.C., Geng, C., Ueda, R., et al. 2006. Staufen- and FMRP-containing neuronal RNPs are structurally and functionally related to somatic P bodies. Neuron 52: 997-1009.

Bashirullah, A., Cooperstock, R.L., and Lipshitz, H.D. 1998. RNA localization in development. Annu. Rev. Biochem. 67: 335-394.

Brendel, C., Rehbein, M., Kreienkamp, H.J., Buck, F., Richter, D., and Kindler, S. 2004. Characterization of Staufen 1 ribonucleoprotein complexes. Biochem. J. 384: 239-246.

Brennan, C.M. and Steitz, J.A. 2001. HuR and mRNA stability. Cell. Mol. Life Sci. 58: 266-277.

Brown, V., Jin, P., Ceman, S., Darnell, J.C., O’Donnell, W.T., Tenenbaum, S.A., Jin, X., Feng, Y., Wilkinson, K.D., Keene, J.D., et al. 2001. Microarray identification of FMRP-associated brain mRNAs and altered mRNA translational profiles in fragile $\mathrm{X}$ syndrome. Cell 107: 477-487.

Buchner, G., Bassi, M.T., Andolfi, G., Ballabio, A., and Franco, B. 1999. Identification of a novel homolog of the Drosophila staufen protein in the chromosome 8q13-q21.1 region. Genomics 62: $113-118$.

Chekulaeva, M., Hentze, M.W., and Ephrussi, A. 2006. Bruno acts as a dual repressor of oskar translation, promoting mRNA oligomerization and formation of silencing particles. Cell 124: 521-533.

Chen, C.Y., Gherzi, R., Ong, S.E., Chan, E.L., Raijmakers, R., Pruijn, G.J., Stoecklin, G., Moroni, C., Mann, M., and Karin, M. 2001. AU binding proteins recruit the exosome to degrade AREcontaining mRNAs. Cell 107: 451-464.

Ciechanover, A., Orian, A., and Schwartz, A.L. 2000. Ubiquitinmediated proteolysis: Biological regulation via destruction. Bioessays 22: 442-451.

Dennis Jr., G., Sherman, B.T., Hosack, D.A., Yang, J., Gao, W., Lane, H.C., and Lempicki, R.A. 2003. DAVID: Database for Annotation, Visualization, and Integrated Discovery. Genome Biol. 4: 3. doi: 10.1186/gb-2003-4-5-p3.

Duchaine, T., Wang, H.J., Luo, M., Steinberg, S.V., Nabi, I.R., and DesGroseillers, L. 2000. A novel murine Staufen isoform modulates the RNA content of Staufen complexes. Mol. Cell. Biol. 20: 5592-5601.

Duchaine, T.F., Hemraj, I., Furic, L., Deitinghoff, A., Kiebler, M.A., and DesGroseillers, L. 2002. Staufen2 isoforms localize to the somatodendritic domain of neurons and interact with different organelles. J. Cell Sci. 115: 3285-3295.

Dugre-Brisson, S., Elvira, G., Boulay, K., Chatel-Chaix, L., Mouland, A.J., and DesGroseillers, L. 2005. Interaction of Staufen1 with the $5^{\prime}$ end of mRNA facilitates translation of these RNAs. Nucleic Acids Res. 33: 4797-4812. doi: 10.1093/nar/gki794.

Elvira, G., Massie, B., and DesGroseillers, L. 2006a. The zinc-finger protein ZFR is critical for Staufen 2 isoform specific nucleocytoplasmic shuttling in neurons. J. Neurochem. 96: 105-117.

Elvira, G., Wasiak, S., Blandford, V., Tong, X.K., Serrano, A., Fan, X., del Rayo Sanchez-Carbente, M., Servant, F., Bell, A.W., Boismenu, D., et al. 2006b. Characterization of an RNA granule from developing brain. Mol. Cell. Proteomics 5: 635-651.

Evdokimova, V., Ruzanov, P., Anglesio, M.S., Sorokin, A.V., Ovchinnikov, L.P., Buckley, J., Triche, T.J., Sonenberg, N., and Sorensen, P.H. 2006. Akt-mediated YB-1 phosphorylation activates translation of silent mRNA species. Mol. Cell. Biol. 26: 277-292.

Ferrandon, D., Elphick, L., Nusslein-Volhard, C., and St Johnston, D. 1994. Staufen protein associates with the 3' UTR of bicoid mRNA to form particles that move in a microtubule-dependent manner. Cell 79: 1221-1232. 
Fonseca, R., Vabulas, R.M., Hartl, F.U., Bonhoeffer, T., and Nagerl, U.V. 2006. A balance of protein synthesis and proteasomedependent degradation determines the maintenance of LTP. Neuron 52: 239-245.

Gama-Carvalho, M., Barbosa-Morais, N.L., Brodsky, A.S., Silver, P.A., and Carmo-Fonseca, M. 2006. Genome-wide identification of functionally distinct subsets of cellular mRNAs associated with two nucleocytoplasmic-shuttling mammalian splicing factors. Genome Biol. 7: R113. doi: 10.1186/gb-2006-7-11-r113.

Hallegger, M., Taschner, A., and Jantsch, M.F. 2006. RNA aptamers binding the double-stranded RNA-binding domain. RNA 12: 1993-2004.

Hegde, A.N., Inokuchi, K., Pei, W., Casadio, A., Ghirardi, M., Chain, D.G., Martin, K.C., Kandel, E.R., and Schwartz, J.H. 1997. Ubiquitin C-terminal hydrolase is an immediate-early gene essential for long-term facilitation in Aplysia. Cell 89: 115-126.

Herbert, A. and Rich, A. 2001. The role of binding domains for dsRNA and Z-DNA in the in vivo editing of minimal substrates by ADAR1. Proc. Natl. Acad. Sci. 98: 12132-12137.

Huttelmaier, S., Zenklusen, D., Lederer, M., Dictenberg, J., Lorenz, M., Meng, X., Bassell, G.J., Condeelis, J., and Singer, R.H. 2005. Spatial regulation of $\beta$-actin translation by Src-dependent phosphorylation of ZBP1. Nature 438: 512-515.

Jonson, L., Vikesaa, J., Krogh, A., Nielsen, L.K., Hansen, T.V., Borup, R., Johnsen, A.H., Christiansen, J., and Nielsen, F.C. 2007. Molecular composition of IMP1 RNP granules. Mol. Cell. Proteomics 6: 798-811.

Kanai, Y., Dohmae, N., and Hirokawa, N. 2004. Kinesin transports RNA: Isolation and characterization of an RNA-transporting granule. Neuron 43: 513-525.

Karpova, A., Mikhaylova, M., Thomas, U., Knopfel, T., and Behnisch, T. 2006. Involvement of protein synthesis and degradation in long-term potentiation of Schaffer collateral CA1 synapses. J. Neurosci. 26: 4949-4955.

Keene, J.D. and Tenenbaum, S.A. 2002. Eukaryotic mRNPs may represent posttranscriptional operons. Mol. Cell 9: 1161-1167.

Kiebler, M.A., Hemraj, I., Verkade, P., Kohrmann, M., Fortes, P., Marion, R.M., Ortin, J., and Dotti, C.G. 1999. The mammalian staufen protein localizes to the somatodendritic domain of cultured hippocampal neurons: Implications for its involvement in mRNA transport. J. Neurosci. 19: 288-297.

Kim, K.C. and Kim, H.K. 2006. Role of Staufen in dendritic mRNA transport and its modulation. Neurosci. Lett. 397: 48-52.

Kim, Y.K., Furic, L., DesGroseillers, L., and Maquat, L.E. 2005. Mammalian Staufen1 recruits Upf1 to specific mRNA 3' UTRs so as to elicit mRNA decay. Cell 120: 195-208.

Kim, Y.K., Furic, L., Parisien, M., Major, F., DesGroseillers, L., and Maquat, L.E. 2007. Staufen1 regulates diverse classes of mammalian transcripts. EMBO J. 26: 2670-2681.

Kohrmann, M., Luo, M., Kaether, C., DesGroseillers, L., Dotti, C.G., and Kiebler, M.A. 1999. Microtubule-dependent recruitment of
Staufen-green fluorescent protein into large RNA-containing granules and subsequent dendritic transport in living hippocampal neurons. Mol. Biol. Cell 10: 2945-2953.

Micklem, D.R., Adams, J., Grunert, S., and St Johnston, D. 2000. Distinct roles of two conserved Staufen domains in oskar mRNA localization and translation. EMBO J. 19: 1366-1377.

Ohashi, S., Koike, K., Omori, A., Ichinose, S., Ohara, S., Kobayashi, S., Sato, T.A., and Anzai, K. 2002. Identification of mRNA/protein (mRNP) complexes containing Pur $\alpha, \mathrm{mStaufen}$, fragile $\mathrm{X}$ protein, and myosin $\mathrm{Va}$ and their association with rough endoplasmic reticulum equipped with a kinesin motor. J. Biol. Chem. 277: 37804-37810.

Ramos, A., Bayer, P., and Varani, G. 1999. Determination of the structure of the RNA complex of a double-stranded RNAbinding domain from Drosophila Staufen protein. Biopolymers 52: 181-196.

Ramos, A., Grunert, S., Adams, J., Micklem, D.R., Proctor, M.R., Freund, S., Bycroft, M., St Johnston, D., and Varani, G. 2000. RNA recognition by a Staufen double-stranded RNA-binding domain. EMBO J. 19: 997-1009.

Riechmann, V. and Ephrussi, A. 2001. Axis formation during Drosophila oogenesis. Curr. Opin. Genet. Dev. 11: 374-383.

Tang, S.J., Meulemans, D., Vazquez, L., Colaco, N., and Schuman, E. 2001. A role for a rat homolog of Staufen in the transport of RNA to neuronal dendrites. Neuron 32: 463-475.

Thomas, M.G., Martinez Tosar, L.J., Loschi, M., Pasquini, J.M., Correale, J., Kindler, S., and Boccaccio, G.L. 2005. Staufen recruitment into stress granules does not affect early mRNA transport in oligodendrocytes. Mol. Biol. Cell 16: 405-420.

Townley-Tilson, W.H., Pendergrass, S.A., Marzluff, W.F., and Whitfield, M.L. 2006. Genome-wide analysis of mRNAs bound to the histone stem-loop binding protein. RNA 12: 18531867.

Vagner, S., Galy, B., and Pyronnet, S. 2001. Irresistible IRES. Attracting the translation machinery to internal ribosome entry sites. EMBO Rep. 2: 893-898.

Villace, P., Marion, R.M., and Ortin, J. 2004. The composition of Staufen-containing RNA granules from human cells indicates their role in the regulated transport and translation of messenger RNAs. Nucleic Acids Res. 32: 2411-2420. doi: 10.1093/nar/ gkh552.

Wickham, L., Duchaine, T., Luo, M., Nabi, I.R., and DesGroseillers, L. 1999. Mammalian Staufen is a double-stranded RNA- and tubulin-binding protein which localizes to the rough endoplasmic reticulum. Mol. Cell. Biol. 19: 2220-2230.

Wodicka, L., Dong, H., Mittmann, M., Ho, M.H., and Lockhart, D.J. 1997. Genome-wide expression monitoring in Saccharomyces cerevisiae. Nat. Biotechnol. 15: 1359-1367.

Zalfa, F., Achsel, T., and Bagni, C. 2006. mRNPs, polysomes, or granules: FMRP in neuronal protein synthesis. Curr. Opin. Neurobiol. 16: 265-269. 

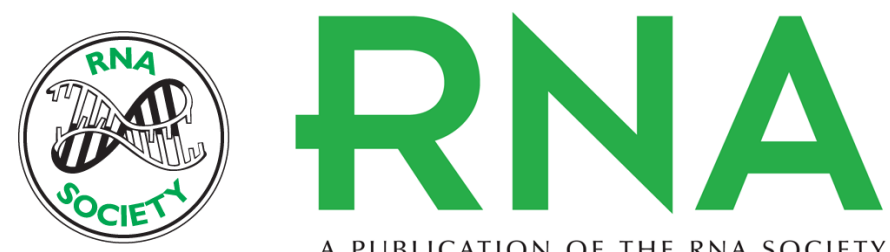

A PUBLICATION OF THE RNA SOCIETY

\section{A genome-wide approach identifies distinct but overlapping subsets of cellular mRNAs associated with Staufen1- and Staufen2-containing ribonucleoprotein complexes}

Luc Furic, Marjolaine Maher-Laporte and Luc DesGroseillers

RNA 2008 14: 324-335

Supplemental http://rnajournal.cshlp.org/content/suppl/2007/12/19/rna.720308.DC1
Material

References This article cites 46 articles, 17 of which can be accessed free at:

http://rnajournal.cshlp.org/content/14/2/324.full.htmI\#ref-list-1

License

Email Alerting Receive free email alerts when new articles cite this article - sign up in the box at the

Service top right corner of the article or click here. 